\title{
La dualidad en el relato «La noche boca arriba» de Julio Cortázar desde la gramática pedagógica
}

ISABEL PAUlo SELVI

IES Els Évols

46021320@edu.gva.es

\begin{abstract}
Resumen: La enseñanza de la gramática en el aula de ELE no está exenta de controversia y de dificultades. El estudiante de ELE necesita una instrucción formal, una enseñanza gramatical explícita que le permita interiorizar las normas y convertir ese conocimiento explícito en implícito.

El relato de Cortázar propone una transgresión temporal (un juego con analepsis y prolepsis), dentro de una estructura narrativa que se desarrolla a través de dos dé́cticos temporales -aquí / allá- y una compleja red de morfología verbal. Este artículo ofrece actividades realizadas desde la perspectiva de una gramática pedagógica, en la que el alumno (con un dominio lingüístico de nivel alto) participa activamente en su proceso de aprendizaje, para lograr una mejor comprensión de los tiempos verbales, uno de los problemas gramaticales recurrentes en el aula de ELE.
\end{abstract}

Palabras clave: La noche boca arriba, Cortázar, gramática pedagógica, ELE.

The duality in the story La noche boca arriba by Julio Cortázar from pedagogical grammar

Abstract: The teaching of grammar in the ELE classroom is not without controversy and difficulties. The ELE student needs a formal instruction, an explicit grammar teaching that allows him to internalize the rules and make that explicit knowledge implicit.

Cortazar's story proposes a temporary transgression (a game with analepsis and prolepsis), within a narrative structure that develops through two temporary deictics here / there - and a complex network of verbal morphology. This article offers activities carried out from the perspective of a pedagogical grammar, in which the student actively participates in their learning process, to achieve a better understanding of the tenses, one of the recurring grammatical problems in the ELE classroom.

Key words: Cortazar's story, pedagogical grammar, ELE.

\section{Enseñar gramática en el aula de ELE}

Una de las preguntas que surge cuando se intenta enseñar gramática en el aula de ELE es qué tipo de gramática y cuánta hay que enseñar. Si tenemos presente la importancia del enfoque gramatical en la enseñanza de ELE desde un punto de vista diacrónico, podemos comprobar de qué manera cada método se ha apoyado en una teoría lingüística 
que ha determinado una manera de enseñar la gramática ${ }^{1}$. La gramática debe enseñarse dentro del enfoque comunicativo y no considerarla fuera de su contexto, porque se corre el riesgo de desvirtuarla, analizándola entonces como una serie de formas lingüísticas desconectadas o independientes. Se trataría de diferenciar entre un conocimiento instrumental de la gramática y un conocimiento declarativo (Pastor Cesteros, 2005: 389).

¿Se pone el foco en la forma o en las formas? Esta es otra de las preguntas que tenemos que solucionar si queremos que la gramática deje de ser meramente descriptiva. Poner el "foco en la forma" (Ellis, 2001) es un principio metodológico basado en tareas que focalizan determinados elementos presentados en el mismo momento en que los discentes planteen los problemas. En este sentido, se favorecen técnicas implícitas hacia determinados rasgos formales del input, no porque estos sean elementos gramaticales aislados sino porque el aprendiente advierte la forma y el uso de tales elementos gramaticales dentro de un contexto. El "foco en la forma" establece una relación cognitiva entre el aprendiente y la forma lingüística, la cual permite un procesamiento basado en la percepción y no en la memorización (Long \& Robinson, 1998: 23). El segundo, el "foco en las formas", trata las formas gramaticales como elementos separados de significado y de su contexto.

Según Martín Peris (1998: 8), el enfoque comunicativo y la enseñanza de la gramática es fundamental en el aula de ELE, y también es necesario incorporar todas las reglas del sistema y del uso lingüístico. En este sentido, para desarrollar la competencia gramatical del discente es aconsejable el uso de una gramática pedagógica.

Con la gramática cognitiva ${ }^{2}$ es posible encontrar un camino para que los estudiantes aprendan de manera más natural, con una adecuada selección de contenidos gramaticales y con un nuevo enfoque del tratamiento del error. El modelo de la gramática cognitiva, desarrollado por R. W. Langacker (1987, 1991, 2001, 2008, 2009), se caracteriza por una serie de rasgos (que lo distinguen de otras propuestas tanto formalistas como funcionales), que lo convierten en un punto de partida tanto teórico (gramática pedagógica) como práctico (enfoque comunicativo).

En una entrevista realizada a Ruíz Campillo en la revista Marcoele sobre la enseñanza de la gramática en el aula, el autor comentaba las pautas a seguir para llevar a la práctica

\footnotetext{
${ }^{1}$ Así, por ejemplo, en el método de gramática-traducción se primaba una teoría lingüística tradicional donde se prevalecía la importancia de la grafía y la palabra; desde una perspectiva normativa se tenía en cuenta la destreza oral y escrita desde el punto de vista de la corrección lingüística; desde una perspectiva deductiva se tenía en cuenta la estructura y la ejemplificación de la misma; desde una perspectiva explícita donde se ofrecen las reglas gramaticales con toda la terminología metalingüística. En el método audio-oral, la gramática era descriptiva (el uso y no tanto la corrección de la norma), implícita (sin la presentación de las reglas) e inductiva (a partir de la práctica se llegaba a interiorizar la teoría).

${ }^{2}$ Esta disciplina nace en los años '70 del siglo XX y plantea un acercamiento diferente al presentado por otros modelos, especialmente generativistas. Podríamos considerar que hay una relación entre la forma, el significado y la conceptualización, pero desde un punto de vista cognitivo, la forma está supeditada al significado e influenciada por el pensamiento del hablante. La gramática pedagógica de ELE se caracteriza por facilitar la comprensión y el dominio de la lengua, para ello efectúa una selección de contenidos que se guía por estos criterios: actualidad, descripción, frecuencia, relevancia comunicativa y otros fenómenos adicionales a los que ofrecen las gramáticas normativas para hablantes nativos.
} 
este enfoque. Se basaba en tres "movimientos de conciencia": del objetivismo al experiencialismo (donde la lengua representa la realidad tal y como la percibe el sujeto y quiere que la perciba el oyente), de la forma al significado (donde la gramática no opera sobre las formas sino sobre el significado con que el hablante usa esas formas en cada momento), de la norma al uso (las manifestaciones periféricas y supuestamente incorrectas dan pistas sobre la gramática).

En una gramática operativa, significativa, o cognitiva, el planteamiento cambia radicalmente, porque la gramática así entendida está indisolublemente unida a la comunicación, la define, la crea, la dirige, la transforma. Si tomamos, pues, "gramática", en este sentido, el tipo de contexto de enseñanza en que nos hallemos será indistinto: en todos los casos la gramática es generadora de la lengua, desde el nivel formal al nivel sintáctico, desde el nivel sintáctico al discursivo, y de ahí al evento de la comunicación real que se produce tanto en la calle como en la pequeña sociedad del aula (Ruíz Campillo, 2007: 8).

Asimismo, el estudio de Reyes Llopis aboga por esta gramática operativa:

La Gramática cognitiva concibe el lenguaje como una representación simbólica del mundo y por tanto su gramática está estrechamente relacionada con la realidad, reflejándola y ayudando a la transmisión de significado a través de sus formas. Esto quiere decir que la gramática es el vehículo de comunicación y no un listado de varias formas para una función o de funciones que admiten diferentes formas y que se practican "comunicativamente" en el aula, aunque sin darle el valor necesario a su significado (Llopis García, 2011: 102).

En esta propuesta, las categorías lingüísticas se entienden como categorías complejas en las que se integran valores generales y prototípicos con significados básicos y secundarios. Para ello, optamos por una gramática operativa que permita al aprendiente ser consciente de la naturaleza del modo verbal y de los procesos comunicativos.

\section{EI espacio como marco operativo}

Teniendo presente todo lo expuesto hasta ahora, es evidente que desde el punto de vista metodológico puede resultar frustrante este laberinto de formas, significados y relaciones de los tiempos y modos verbales. Fijémonos en los valores del imperfecto ${ }^{3}$ :

a. Valor prototípico (pasado no terminado): "Me lo encontré cuando bajaba al portal".

b. Situación hipotética (no situado en el pasado y alternando con el condicional): "Si pudiera, me compraba un coche".

c. Extensiones de valor prototípico de pasado a valores contrafactuales, que comparten con el sentido del pasado la pertenencia a un ámbito no actual (valor esquemático del presente de pasado, terminativo o no terminativo): "En ese momento, Pedro ganaba el premio de la lotería".

Para Castañeda et al. (2014) la relación entre el valor esquemático y los valores prototípico y extendido es como la relación entre un hiperónimo (presente de pasado) y dos hipónimos (pasado no terminado y valor contrafactual). Ruíz Campillo (2007) propone un desplazamiento que vaya de la instrucción descriptiva a un análisis de los significados atribuidos a una forma. En este sentido, habla de una visión del modo verbal que parta de un valor de operación (una gramática operativa), es decir, "una

\footnotetext{
${ }^{3}$ Las referencias de marco teórico están basadas en la obra coordinada por Alejandro Castañeda (2014), con ejemplificaciones diferentes, adaptadas a la propuesta que presentamos.
} 
gramática de significados básicos e inalterables con los que tomar decisiones gramaticales" (Ruíz Campillo, 2007 a: 6). Esta gramática no puede estar basada en listas de reglas no funcionales sino en elementos comunes que tengan los aprendientes ya en su propia lengua ${ }^{4}$.

La gramática operativa puede resolver de manera "natural" los problemas siempre planteados entre el presente perfecto (pretérito perfecto) e indefinido. El primero definido como "acción que está concluida y que el hablante sitúa, discursivamente, cerca de su espacio inmediato", y el indefinido como "acción concluida" (LLopis \&Ruiz 2012: 86). El hablante se sirve de estos valores para señalar una actitud lingüística y su diferencia estriba en la marca y grado de actualidad que el mismo hablante otorga. Además, tanto Llopis como Ruíz critican la costumbre de enseñar estas formas verbales recurriendo a los marcadores temporales "hoy / ayer", como si todo el sistema pudiera realizarse con el perfecto o con el indefinido de una manera esquematizada.

El que aparezca de una forma u otra es algo circunstancial y no el resultado de una ley explícita, porque "que una acción haya tenido lugar hoy no la convierte en necesariamente relevante para el hablante, y que haya ocurrido ayer, no necesariamente la hace perder su rasgo de relevancia y actualidad" (Llopis García \& Ruíz Campillo, 2012: 87). Como dilucidar esto no es una tarea fácil, estos autores recomiendan el uso del indefinido y aconsejan el uso del presente perfecto cuando el estudiante perciba que su uso está plenamente justificado.

Otro de los problemas viene del contraste verbal entre el pretérito imperfecto y el indefinido. Para estos autores, el imperfecto ve la escena desde dentro pero su acción no está concluida, mientras que con el indefinido la ve desde fuera y la percibe como un hecho ya terminado. Como se observa, estos valores verbales tienen una base espacial, así tanto el imperfecto como el indefinido expresan un espacio alejado (Llopis García \& Ruíz Campillo, 2012: 170). Este posicionamiento desde dentro y en un espacio alejado es lo que hace que el imperfecto pueda concretarse en otras funciones que no puede tener el indefinido, donde siempre la localización la realiza en pasado.

Ruíz Campillo (2015) propone el valor unificador del espacio como mapa operativo y diseña dos categorizaciones: una categorización de tiempos (presente-pasado-futuro) y otra categorización del modo, aspecto o foco. Es decir, el sistema verbal se entiende mejor en términos espaciales. Esta disponibilidad espacial es la que vamos a seguir con la propuesta didáctica que presentamos.

\section{La dualidad en el relato «La noche boca arriba» de Julio Cortázar}

Los motivos temáticos en el relato surgen del realismo fantástico de la cotidianidad mientras que su organización busca la ruptura con la linealidad lógico-temporal, establece el contraste y juega con la paradoja de la existencia bitemporal del sujeto. El escritor reestructura el mito prehispano a través de la elaboración de dos mundos paralelos, en el que el tránsito del sueño del motociclista lo lleva al mundo azteca a

${ }^{4}$ En Gramática básica del estudiante del español (Ruíz Campillo, 2005) se ejemplifica a través de actividades, y en cómo los aprendientes de ELE piensan en cada una de las situaciones planteadas y toman decisiones. 
través del recurso estilístico sinestésico. La dualidad empieza en el mismo inicio del relato, entrelazándose el espacio y el tiempo.

(1) «Como sueño era curioso porque estaba lleno de olores y él nunca soñaba olores. Primero un olor a pantano, ya que a la izquierda de la calzada empezaban las marismas, los tembladerales de donde no volvía nadie. Pero el olor cesó, y en cambio vino una fragancia compuesta y oscura como la noche en que se movía huyendo de los aztecas» (Cortázar, La noche boca arriba, 1956:161).

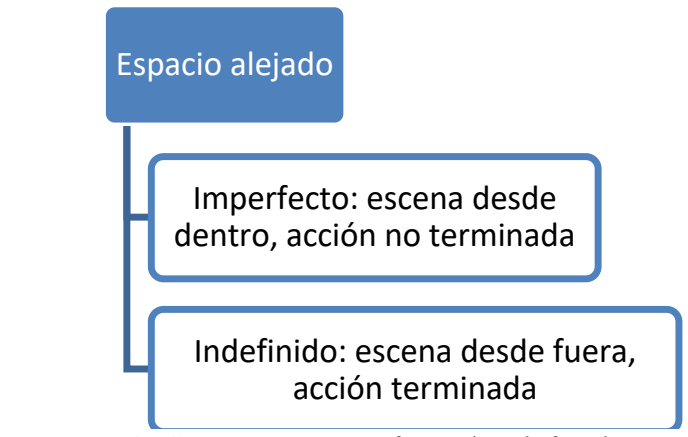

Figura 1. Contraste Imperfecto / Indefinido. Fuente propia.

La historia avanza en una transgresión temporal (juega con la analepsis y la prolepsis), desarrollando una estructura narrativa que podemos focalizar a través de dos deícticos temporales (aqui/ alli). Desde el punto de vista de la gramática pedagógica, con el Presente Perfecto afirmamos hechos terminados en un espacio actual (nos situamos en el aquí), si usamos el Indefinido presentamos el hecho en sí mismo y no en relación con un espacio actual (contamos hechos completos y terminados en el espacio pasado en que se produjeron, es decir, nos situamos después del hecho pasado).

Finalmente, con el Imperfecto describimos hechos no terminados en el momento del pasado o situaciones momentáneas, y en este sentido nos situamos dentro del hecho pasado, en el allí. En el relato de Cortázar, el protagonista no se da cuenta hasta el final del relato de cuál es el sueño y cuál es la realidad, porque el sueño es justamente lo real, es decir, el joven que está a punto de ser sacrificado.

Este juego temporal sorpresivo fija el contraste entre el Indefinido, el Imperfecto y otros tiempos que se organizan. Hay una total ausencia del Presente Perfecto (como espacio cercano al hablante y de acción concluida) para focalizar los valores del Indefinido y del Imperfecto junto con el Pluscuamperfecto, con el objetivo de manifestar una actitud lingüística y un grado de mayor o menor certeza entre la realidad y el sueño. Recordemos que esta ausencia tiene que ver con la decadencia de esa distinción en América Latina, y esto es interesante para señalar la diferencia en el uso de este tiempo en lugares diferentes del mundo hispanohablante.

(2) «Volvió bruscamente del desmayo. Cuatro o cinco hombres jóvenes lo estaban sacando de debajo de la moto. Sentía gusto a sal y sangre, le dolía una rodilla, y cuando lo alzaron gritó, porque no podía soportar la presión en el brazo derecho. Voces que no parecían pertenecer a las caras suspendidas sobre él, lo alentaban con bromas y seguridades. Su único alivio fue oír la confirmación de que había estado en su derecho al cruzar la esquina» (Cortázar, La noche boca arriba, 1956:160). 
(3) «Lo que más lo torturaba era el olor, como si aun en la absoluta aceptación del sueño algo se revelara contra eso que no era habitual, que hasta entonces no había participado del juego. «Huele a guerra», pensó, tocando instintivamente el puñal de piedra atravesado en su ceñidor de lana tejida» (Cortázar, La noche boca arriba, 1956: 162).

(4) «Como dormía de espaldas, no lo sorprendió la posición en que volvía a reconocerse, pero en cambio el olor a humedad, a piedra rezumante de filtraciones, le cerró la garganta y lo obligó a comprender. Inútil abrir los ojos y mirar en todas direcciones; lo envolvía una oscuridad absoluta. Quiso enderezarse y sintió las sogas en las muñecas y los tobillos. Estaba estaqueado en el suelo, en un piso de lajas helado y húmedo» (Cortázar, La noche boca arriba, 1956: 164).

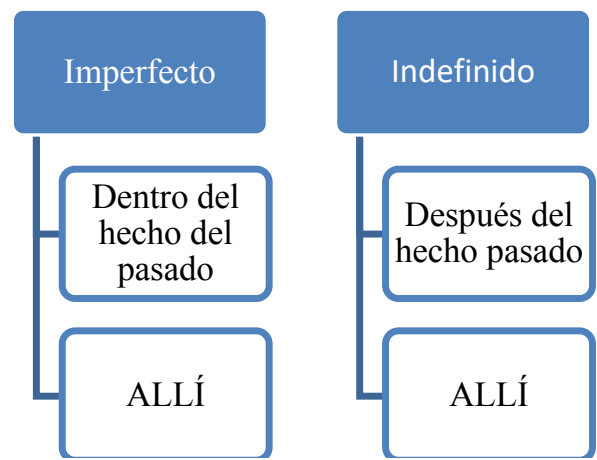

Figura 2. Contraste entre el Imperfecto y el Indefinido. Fuente propia.

Ruíz Campillo (2014) organiza las formas verbales en dos espacios (actual e inactual) y tres dimensiones modales de cada uno de esos espacios (positivo, aproximativo y virtual).

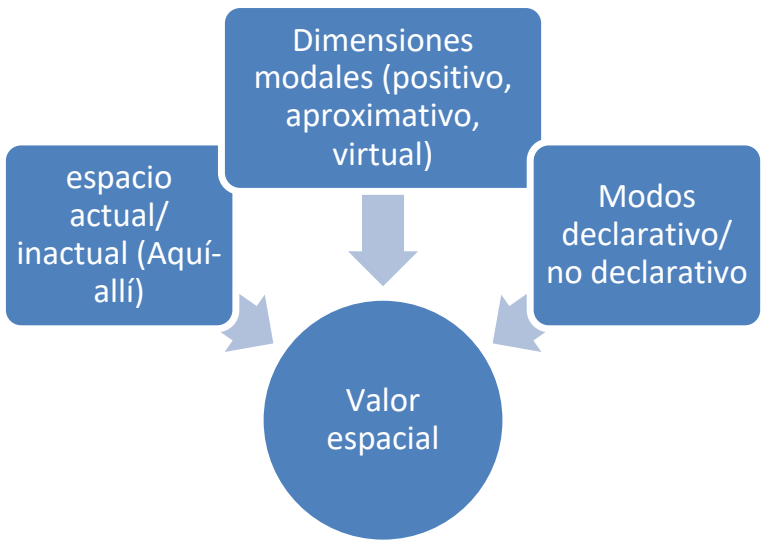

Figura 3. Reorganización del sistema verbal basado en Ruíz Campillo (2014).Fuente propia.

En este espacio del Allí (espacio inactual) se sitúa el Imperfecto (y el Pluscuamperfecto) con un valor positivo (afirmación), el Condicional (y Condicional Compuesto) con un valor de aproximación (predicción) y el Imperfecto (y Pluscuamperfecto) de Subjuntivo como modo no declarativo y espacio virtual. La posición de cada forma determina su significado en relación de correspondencia con las formas con las que comparte espacio (tanto horizontal como vertical). De ello se deduce que cada forma puede tener un solo significado y siempre el mismo. 


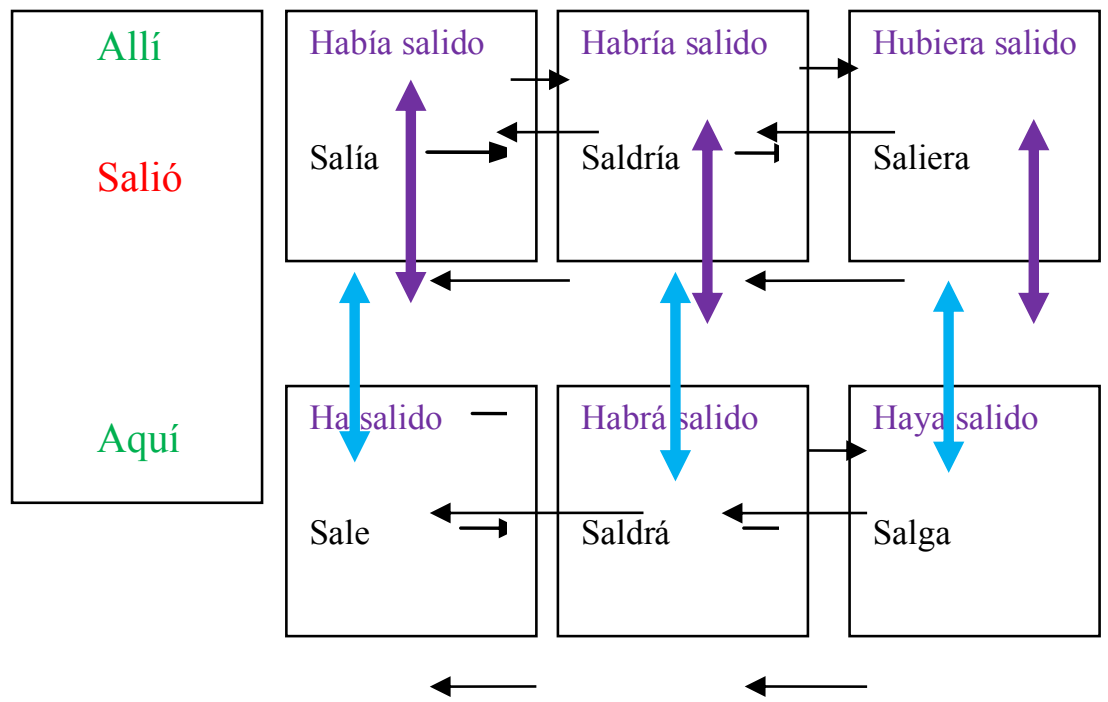

Figura 4. Ley de correspondencia horizontal y vertical. Ruíz Campillo (2014).

En el relato de Cortázar se observa esta recursividad en los tiempos:

Pretérito

Perfecto

simple

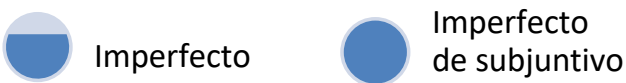

Pluscuamperfect

ALLí

Espacio

inactual

Figura 5. Verbos del relato en su correspondencia. Fuente propia.

En este sentido, tanto la correspondencia vertical como horizontal implica la asimilación significativa del sistema para el discente de ELE. Si se sabe usar el presente, se sabe utilizar el Pretérito Perfecto (Presente Perfecto), porque este es la versión terminativa del presente. El Futuro simple y el compuesto son lo mismo que el Presente y el Perfecto, con la diferencia que presentan una predicción. Sabiendo estos dos pares, se sabe usar el Subjuntivo tanto Presente como Perfecto.

El relato de Cortázar se focaliza desde el Imperfecto hacia el Pluscuamperfecto de Indicativo y Subjuntivo, de ahí que pueda expresar perfectamente la dicotomía temporal y del sujeto protagonista de la acción; sin embargo, el punto de partida lo constituye el Indefinido, la única forma simple de aspecto perfectivo, que tiene el valor de declaración y afirmación. El estudiante de ELE puede ir viendo las correspondencias verticales y horizontales propuestas por Ruíz Campillo a través de la construcción lógica del relato de Cortázar.

En el nivel discursivo, el cuento tiene un final sorpresivo que desmonta las expectativas del lector y provoca la sorpresa; sin embargo, la dicotomía aquí/ allí permanece a través de las correspondencias verbales:

(5) «Pero olía la muerte, y cuando abrió los ojos vio la figura ensangrentada del sacrificador que venía hacia él con el cuchillo de piedra en la mano. Alcanzó a cerrar otra vez los párpados, 
aunque ahora sabía que no iba a despertarse, que estaba despierto, que el sueño maravilloso había sido el otro, absurdo como todos los sueños; un sueño en el que había andado por extrañas avenidas de una ciudad asombrosa, con luces verdes y rojas que ardían sin llama ni humo, con un enorme insecto de metal que zumbaba bajo sus piernas. En la mentira de ese sueño también lo habían alzado del suelo, también alguien se le había acercado con un cuchillo en la mano, a él tendido boca arriba, a él boca arriba con los ojos cerrados entre las hogueras», (Cortázar, La noche boca arriba,1956:164).

\section{Indefinido}

Imperfecto

-Pluscuamperfe

cto
Allí

-Aquí

Figura 6. Final del relato jugando con los tiempos y espacios. Fuente propia.

Este planteamiento de la gramática pedagógica y operativa puede ser eficaz en el proceso de aprendizaje del discente de ELE, interesado en tener un esquema lógico de los tiempos y ser competente desde el punto de vista discursivo. Es una posibilidad, quizás, menos artificial de presentar los tiempos verbales porque se basa en una gramática que potencia la competencia estratégica del hablante. Ya no estamos hablamos de un sujeto pasivo que no interactúa sino de un dinamizador, dando y transmitiendo información, manifestando deseos, sentimientos y opiniones, interpretando roles, y en definitiva, ejercitando su competencia comunicativa.

\section{Conclusiones}

Partiendo de todo lo expuesto y a modo de breve conclusión, nos gustaría insistir en la importancia y en la necesidad de difundir una gramática pedagógica para los discentes de ELE. Sería conveniente que esta gramática procurara también atender a los diferentes niveles del alumnado, proporcionando un input adecuado y favoreciendo asimismo la construcción creativa de la lengua de aprendizaje, al tiempo que desarrollase una práctica adecuada y contextualizada para los tiempos verbales.

El planteamiento de Ruíz Campillo pone al hablante en el punto de mira, en el foco, para que interiorice la visión del modo verbal como parte de un valor de operación, centrándose en una gramática de creación y no de categorización. Ese valor de operación (declarativo-no declarativo) sería el concepto prototípico a partir del cual se podrían crear las asociaciones.

Entender la gramática desde este punto de vista significa liberarse de los listados de reglas que asignan formas a los significados, y poder pensar que existe una lógica que puede transformar la amalgama de elementos aleatorios.

Las implicaciones de una concepción espacial del lenguaje en la enseñanza de ELE pueden ser positivas, porque muchos de los aspectos intrincados de la gramática (y especialmente la morfología verbal) cobran una mejor accesibilidad. 
El relato de Cortázar trabaja ese planteamiento espacial desde el sistema verbal y desde el desdoblamiento del sujeto. Focaliza inmediatamente el tiempo y el modo verbal, es decir, el relato se desarrolla desde el Imperfecto hacia el Pluscuamperfecto, de ahí que pueda expresar perfectamente la dicotomía temporal a través del sujeto protagonista de la acción. Sin embargo, el punto de partida del sujeto del enunciado lo constituye el Indefinido, la única forma simple de aspecto perfectivo que tiene el valor de declaración y afirmación.

El estudiante de ELE puede ir viendo las correspondencias verticales y horizontales propuestas por Ruíz Campillo a través de la construcción lógica del relato de Cortázar, y de qué manera el espacio lejano (simbolizado por el deíctico "allí") se llena de sentido con el espacio cercano (simbolizado por el deíctico "aquí"), para fundirse en uno solo conforme va avanzando la historia.

La tarea no necesariamente tiene que ir dirigida a un estudiante de ELE con un dominio avanzado de la lengua, porque el relato se puede presentar de manera fragmentada y con un visionado previo. Lo interesante es que entienda la necesidad espacial de la morfología verbal y de qué manera el relato de Cortázar puede servir de base para fomentar la producción escrita del alumnado de ELE y su competencia discursiva.

\section{Bibliografía}

CORTÁZAR, J. (1956). «La noche boca arriba». Final de juego. Madrid: Alfaguara.

ELLIS, R. (2001). Form-Focused Instruction and Second Language Learning. Blackwell Publishers

LANGACKER, R. W. (1987). Foundations of Cognitive Grammar. Volume I: Theoretical Prerequisites. Stanford: Standford University Press.

LANGACKER, R. W. (1991). Foundationes of Cognitive Grammar. Volume II: Descriptive Application. Standford: Standford University Press.

LANGACKER, R. W. (2000). «Estructura de la cláusula en la gramática cognoscitiva», Estudios cognoscitivos del español, número monográfico de la revista RESLA (Asociación Española de Lingüística Aplicada), pp. 19-65.

LANGacker, R. W. (2008). Cognitive Grammar: A basic Introduction. Nueva York: Ofxord University Press.

LANGACKER, R. W. (2009). Investigations in Cognitive Grammar. Berlín- Nueva York: Mouton De Gruyter.

LONG, M. \& RoBInson, P. (1998). «Focus on form. Theory, research, and practice». En Doughty, C. \& Williams, J. (eds.); Focus on Form in Classroom Second Language Acquisition. (pp. 15-41). Cambridge: CUP.

LlOPIS GARCÍA, Reyes (2011). «Gramática cognitiva para la enseñanza del español como lengua extranjera. Un estudio con aprendientes alemanes de español como lengua extranjera», ASELE, n. ${ }^{\circ}$ 4, pp. 1-24.

Llopis García, Reyes \& J. M. ReAl Espinosa \& J. P. Ruíz CAMPILlo (2012). Qué gramática enseñar, qué gramática aprender. Madrid: Colección Biblioteca Edinumen de Didáctica. Claves para la enseñanza del español. 
MARTín PERIS, Ernesto (1998). «Gramática y enseñanza de segundas lenguas», Carabela, $\mathrm{n}^{\circ} 43$, pp. 5-32.

PAstor Cesteros, Susana (2005). «La enseñanza de segundas lenguas». En Ángel López \& Beatriz Gallardo (eds); Conocimiento y lenguaje. (pp. 361-399). Valencia: Publicaciones de la Universidad de Valencia

Ruíz CAMPILlo, José Plácido (2007a). «El verbo como espacio», Revista Nebrija de lingüística aplicada a la enseñanza de las lenguas, pp. 34-67.

Ruíz CAmpillo, José Plácido (2007b). «Entrevista a José Plácido Ruiz Campillo: gramática cognitiva y ELE», MarcoELE, n. ${ }^{\circ}$ 5, pp. 56-78. 